\title{
Hydroxide Ion Carrier for Proton Pump in Bacteriorhodopsin: Primary Proton Transfer
}

\author{
Junichi Ono,,$^{1,2}$ Minori Imai, ${ }^{3}$ Yoshifumi Nishimura, ${ }^{1}$ and Hiromi Nakai ${ }^{1,2,3^{*}}$
}

${ }^{1}$ Waseda Research Institute for Science and Engineering (WISE), Waseda University, 3-4-1 Okubo, Shinjuku, Tokyo 169-8555, Japan

${ }^{2}$ Elements Strategy Initiative for Catalysts \& Batteries (ESICB), Kyoto University, 1-30 Goryo-Ohara, Nishikyo-ku, Kyoto 615-8245, Japan

${ }^{3}$ Department of Chemistry and Biochemistry, School of Advanced Science and Engineering, Waseda University, 3-4-1 Okubo, Shinjuku, Tokyo 169-8555, Japan

*Correspondence: nakai@waseda.jp 

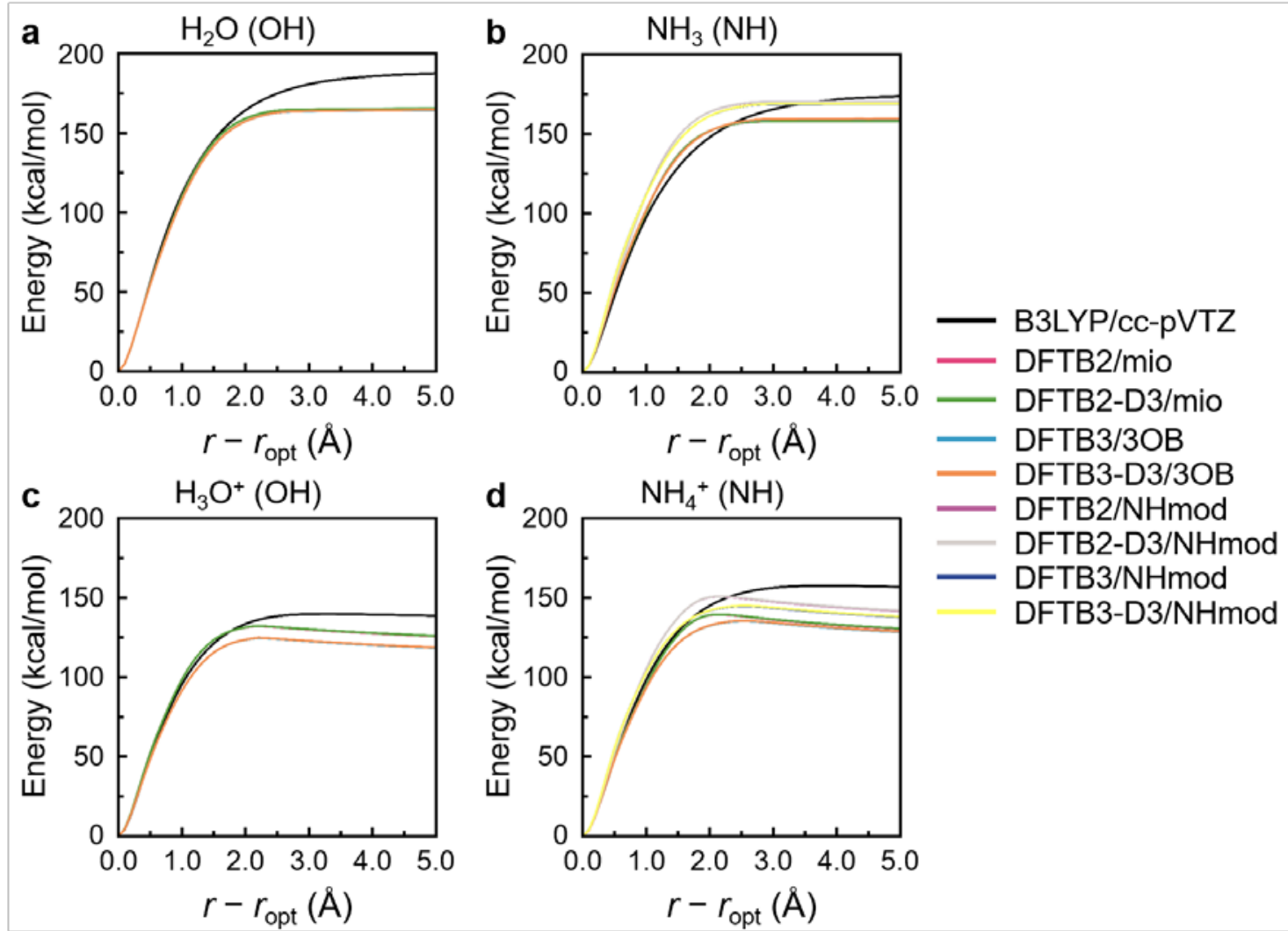

Figure S1. Covalent bond dissociation curves of (a) $\mathrm{H}_{2} \mathrm{O}$, (b) $\mathrm{NH}_{3}$, (c) $\mathrm{H}_{3} \mathrm{O}^{+}$, and (d) $\mathrm{NH}_{4}^{+}$obtained from B3LYP and DFTB calculations. Energies are given as relative value with respect to that of optimized structure $\left(r-r_{\mathrm{opt}}=0.0 \AA\right)$. 


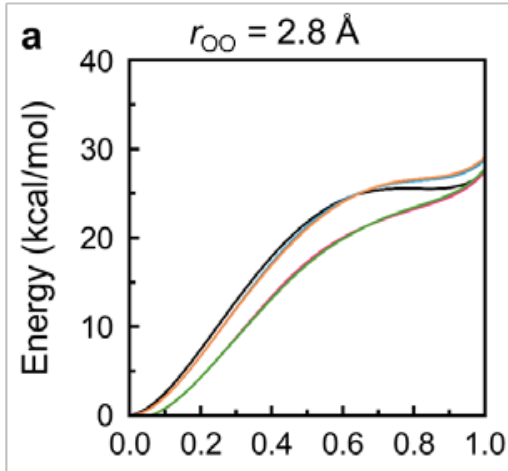

Reaction coordinate

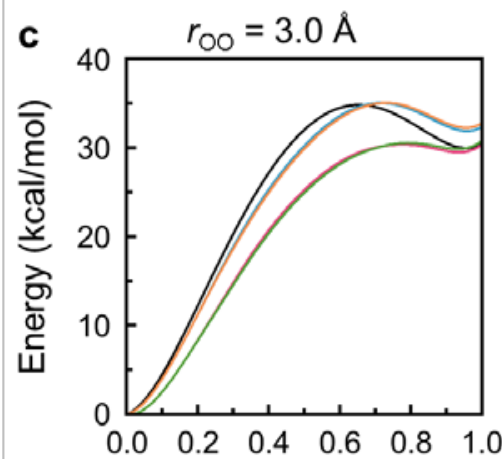

Reaction coordinate
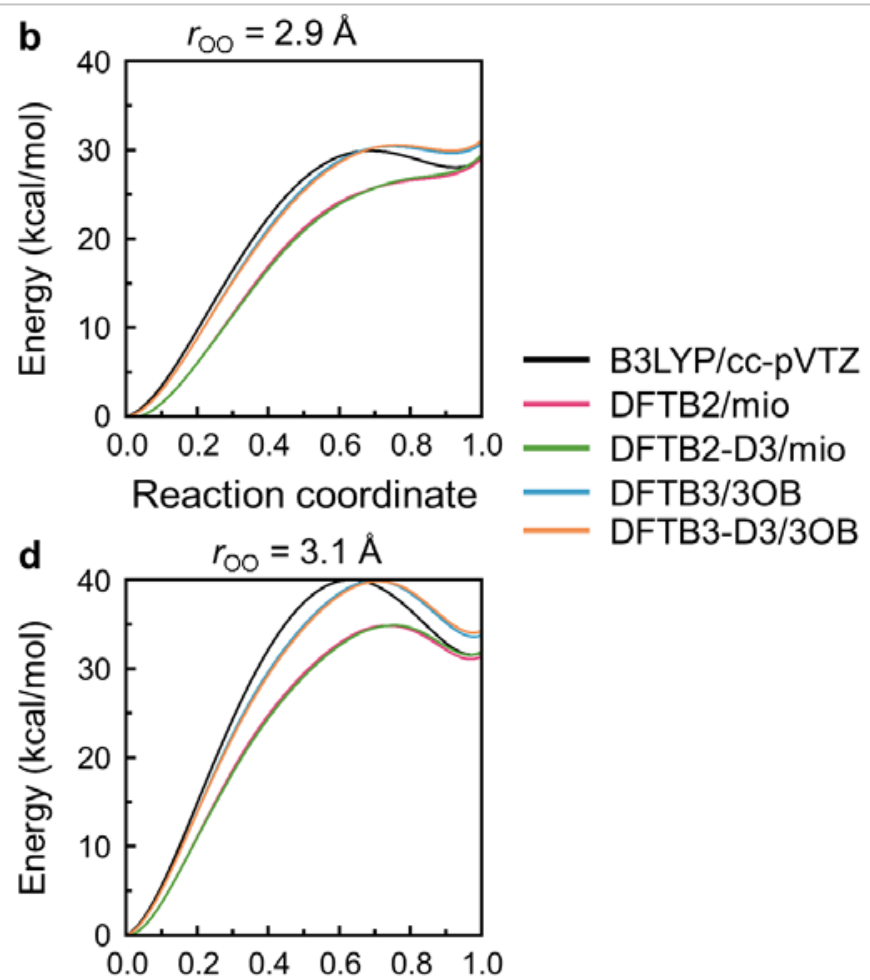

Reaction coordinate
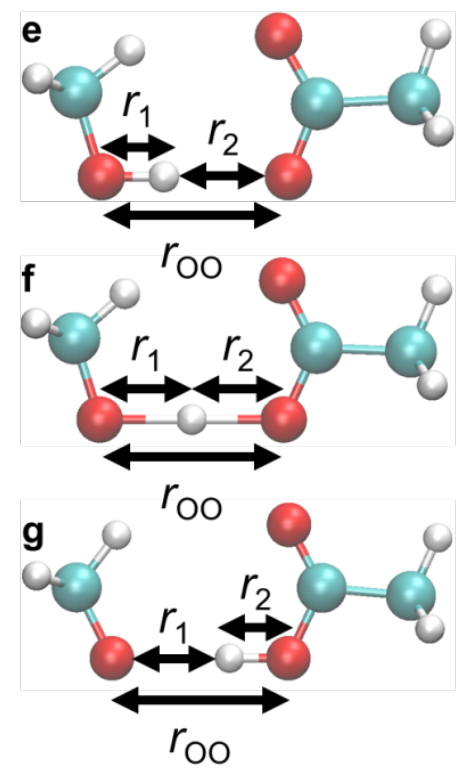

Reaction coordinate $=0.0$ $r_{1}=1.0 \AA, r_{2}=\left(r_{\mathrm{OO}}-1.0\right) \AA$

Reaction coordinate $=0.5$ $r_{1}=r_{2}=0.5 r_{\mathrm{OO}} \AA$

Reaction coordinate $=1.0$ $r_{1}=\left(r_{\mathrm{OO}}-1.0\right) \AA, r_{2}=1.0 \AA$

Figure S2. Potential energy curves for the proton transfer from methanol $(\mathrm{MeOH})$ to acetate $\left(\mathrm{MeCOO}^{-}\right)$ obtained from B3LYP and DFTB calculations with the fixed distances between the donating and accepting oxygen atoms of (a) 2.8, (b) 2.9, (c) 3.0, and (d) $3.1 \AA$. Also shown are the schematic illustrations of the values of the reaction coordinate (RC) for (e) 0.0, (f) 0.5, and (g) 1.0. Energies are given as relative value with respect to that of optimized structure at $\mathrm{RC}=0$. 

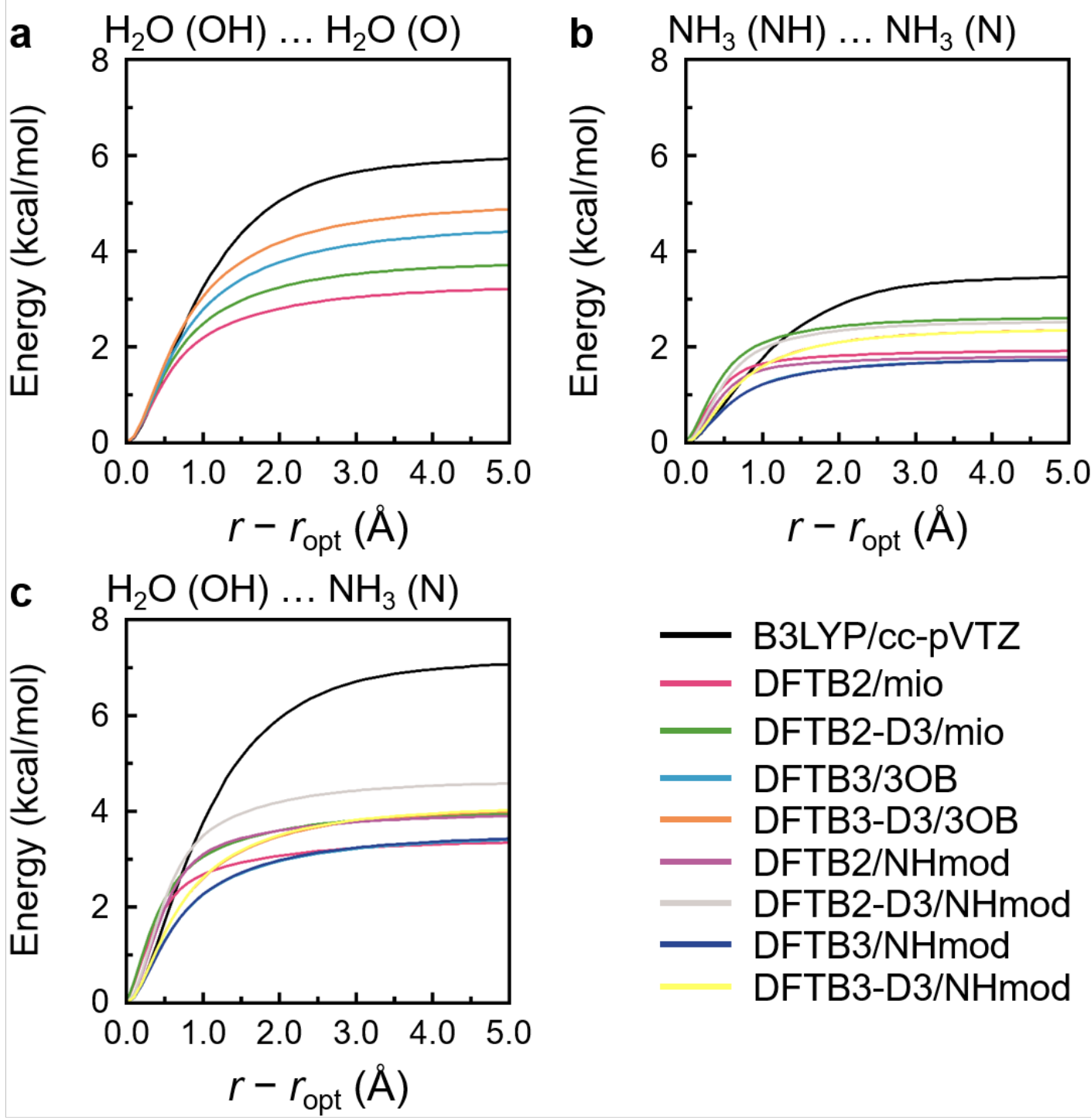

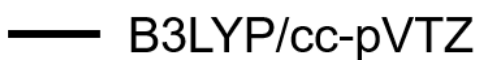
DFTB2/mio DFTB2-D3/mio DFTB3/3OB DFTB3-D3/3OB DFTB2/NHmod DFTB2-D3/NHmod DFTB3/NHmod DFTB3-D3/NHmod

Figure S3. Hydrogen bond dissociation curves of (a) water dimer, (b) ammonia dimer, and (c) water ammonia dimer obtained from B3LYP and DFTB calculations. Energies are given as relative value with respect to that of optimized structure $\left(r-r_{\text {opt }}=0.0 \AA\right)$. 


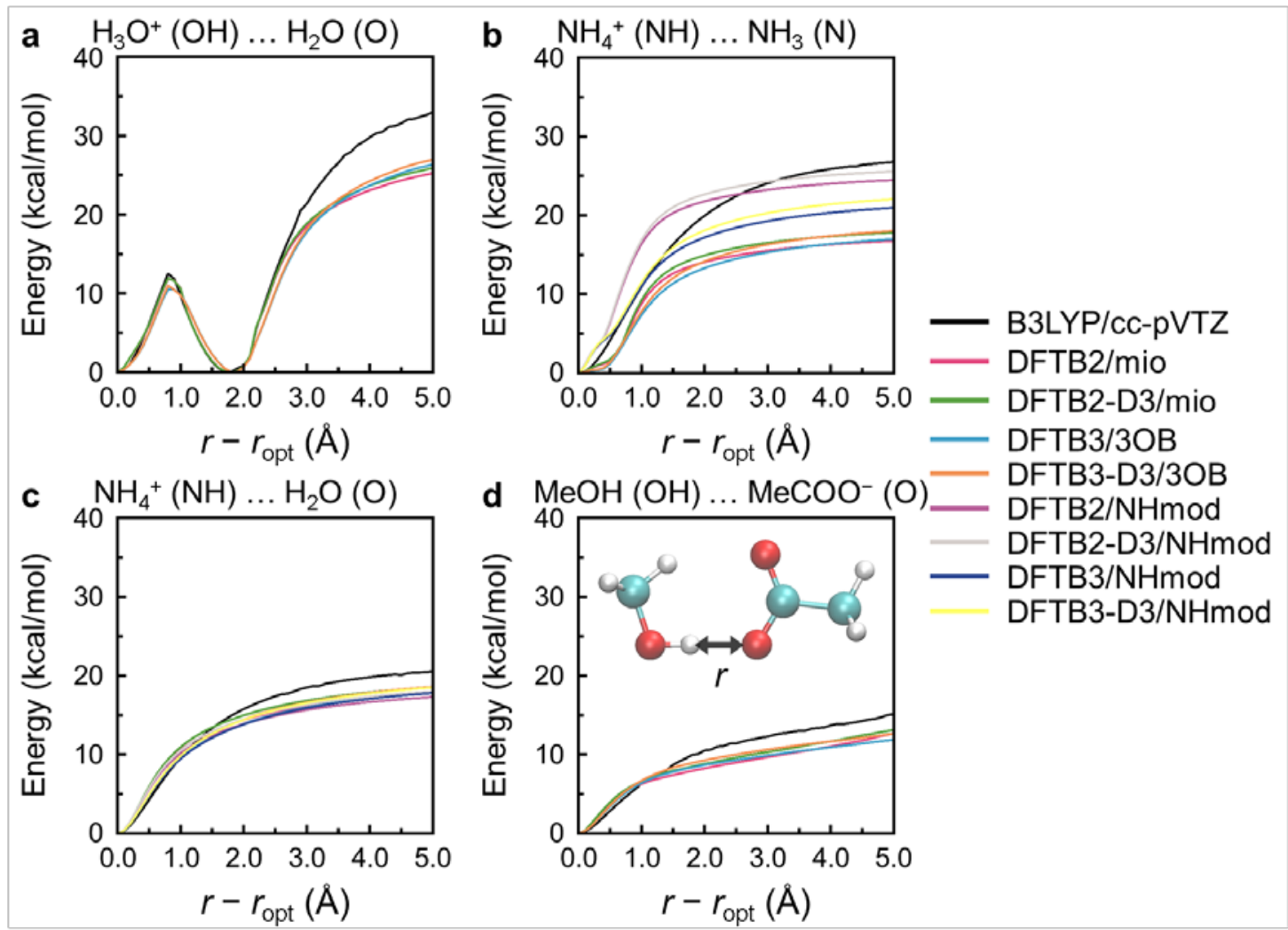

Figure S4. Ionic hydrogen bond dissociation curves of (a) hydronium water dimer, (b) ammonium ammonia dimer, (c) ammonium water dimer, and (d) methanol acetate dimer obtained from B3LYP and DFTB calculations. Energies are given as relative value with respect to that of optimized structure $(r-$ $\left.r_{\mathrm{opt}}=0.0 \AA\right)$. 
(a) Between the $\mathrm{O} / \mathrm{N}$ and $\mathrm{O}$ atoms

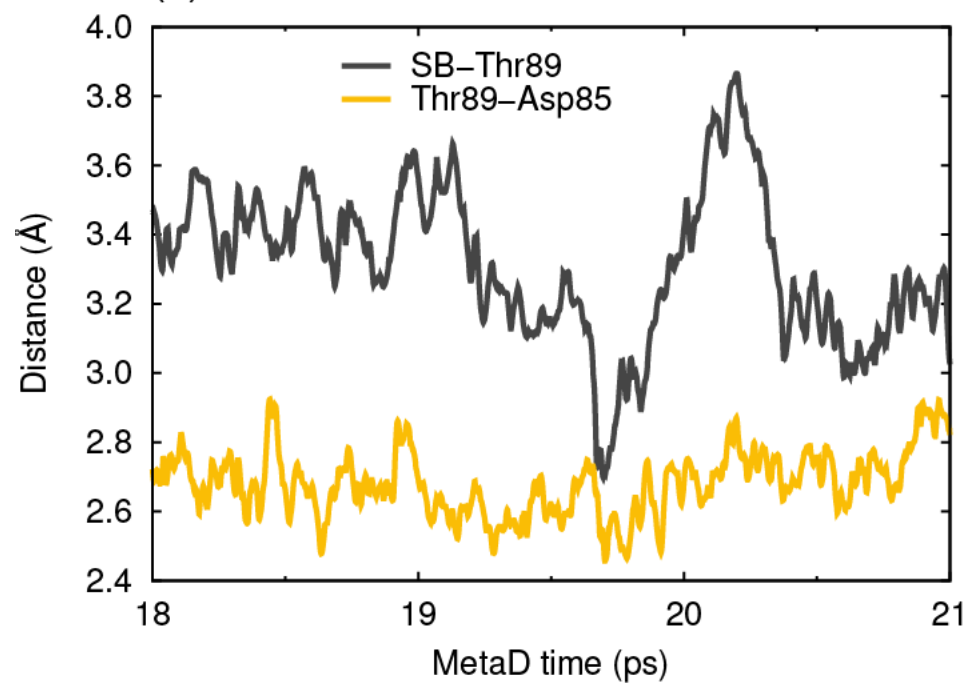

(b) Between the $\mathrm{H}$ and $\mathrm{O}$ atoms

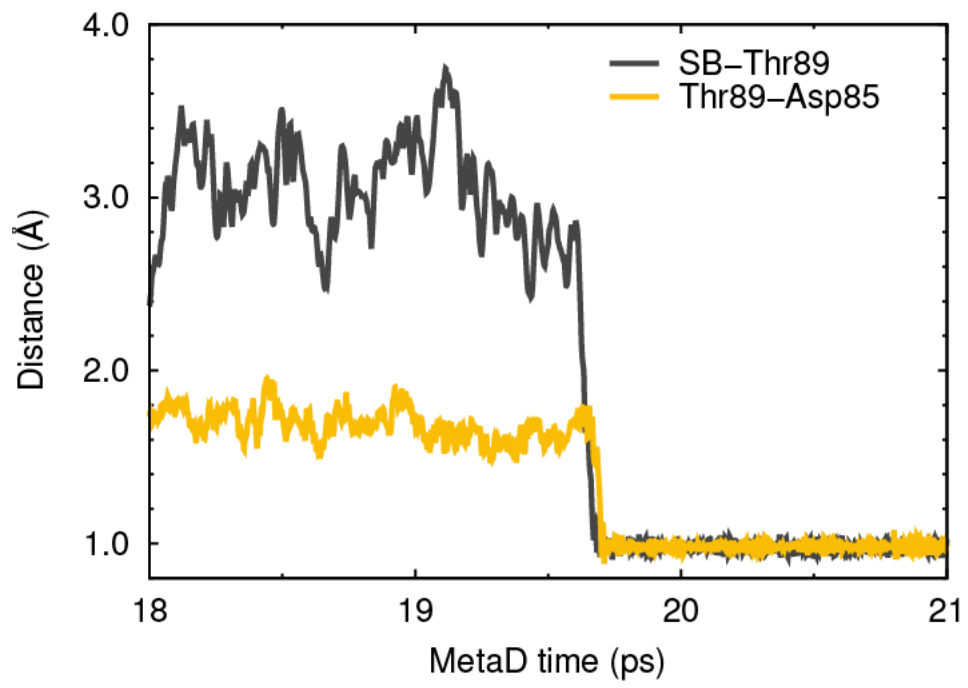

Figure S5. Representative time series of (a) the distances between the $\mathrm{O} / \mathrm{N}$ and $\mathrm{O}$ atoms and (b) those between the $\mathrm{H}$ and $\mathrm{O}$ atoms for the hydrogen-bond pairs in the active site related with the Grotthuss-like proton transfer reactions in path 1 obtained from DC-DFTB-metaD simulations for IBRM starting from the $5 \mathrm{H} 2 \mathrm{~K}$ structure. The deprotonation of SB occurred at 19.6 ps immediately followed by the protonation of Asp85 via Thr89. 
(a) Between the $\mathrm{O} / \mathrm{N}$ and $\mathrm{O}$ atoms

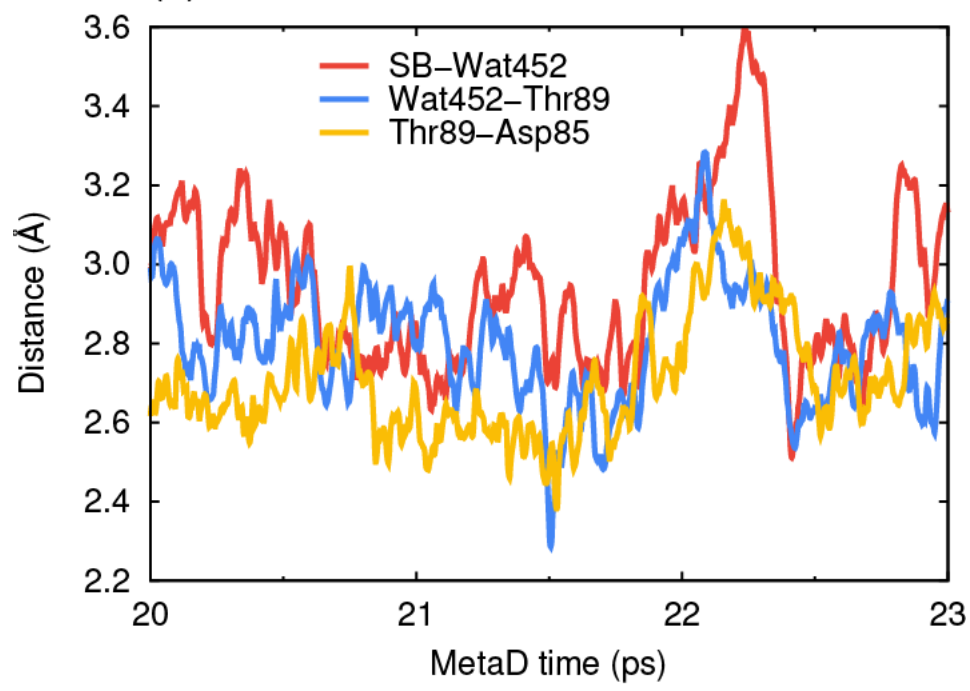

(b) Between the $\mathrm{H}$ and $\mathrm{O}$ atoms

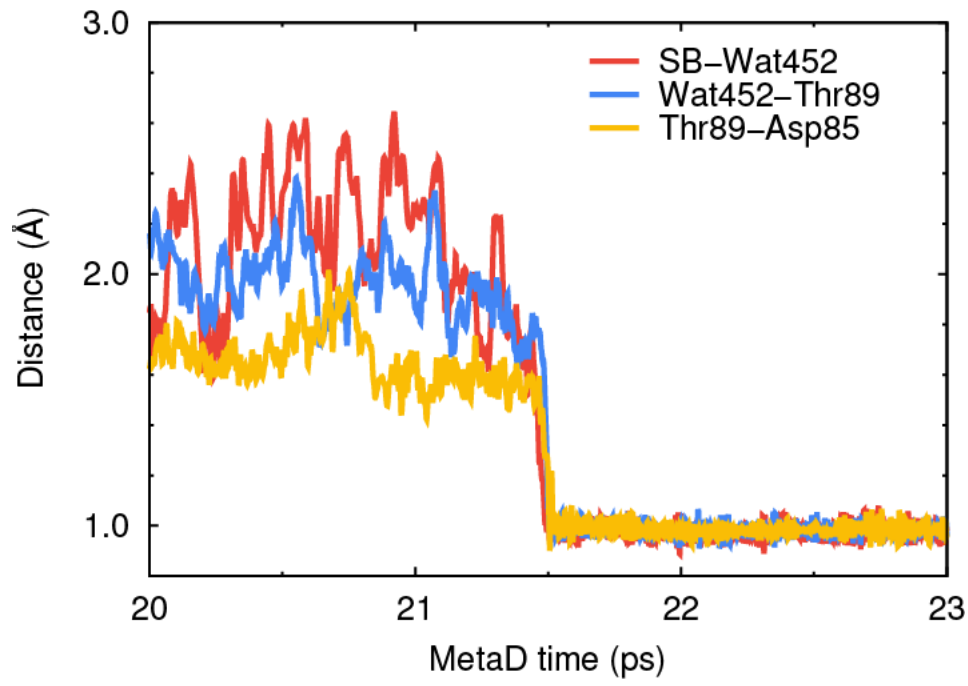

Figure S6. Representative time series of (a) the distances between the $\mathrm{O} / \mathrm{N}$ and $\mathrm{O}$ atoms and (b) those between the $\mathrm{H}$ and $\mathrm{O}$ atoms for the hydrogen-bond pairs in the active site related with the Grotthuss-like proton transfer reactions in path 2 obtained from DC-DFTB-metaD simulations for IBRM starting from the $5 \mathrm{H} 2 \mathrm{~K}$ structure. The deprotonation of SB occurred at $21.5 \mathrm{ps}$ immediately followed by the protonation of Asp85 via Wat452 and Thr89. 

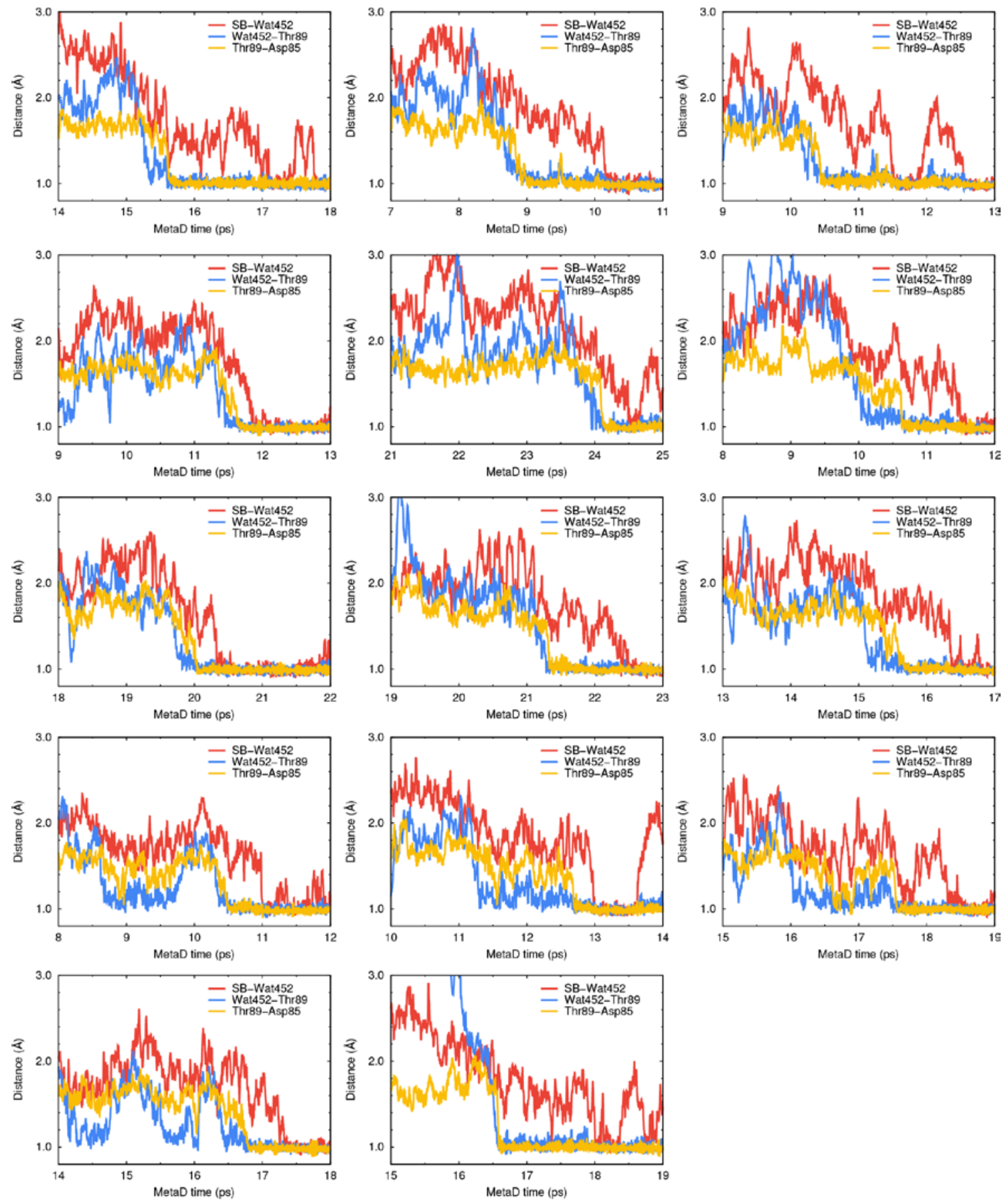

Figure S7. Time series of the distances between the $\mathrm{H}$ and $\mathrm{O}$ atoms for the hydrogen-bond pairs in the active site related with the Grotthuss-like proton transfer reactions in path 3 obtained from 14 DC-DFTBmetaD simulations for IBRM starting from the 5H2K structure. 
Journal of Chemical Physics B

Supporting Information

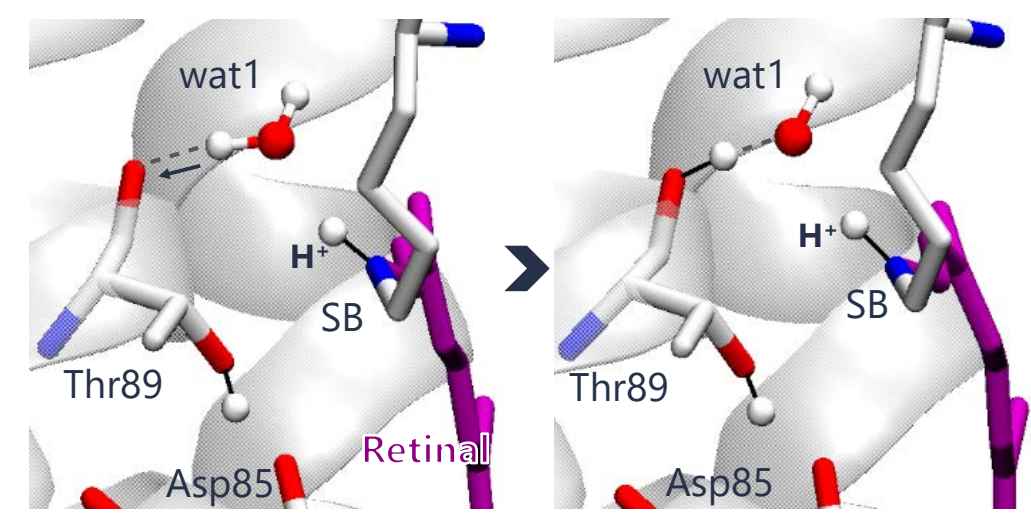

Figure S8. Representative snapshots of the irrelevant proton transfer from SB to the backbone carbonyl group of Thr89 observed in the DC-DFTB-metaD simulations starting from 5H2L or 5H2M. 
(a) $5 \mathrm{~B} 6 \mathrm{X}(760 \mathrm{~ns})$

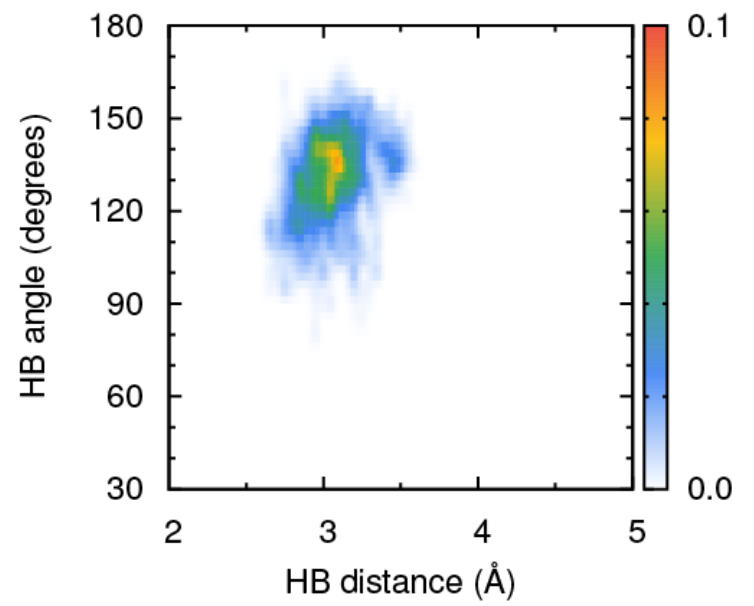

(c) $5 \mathrm{H} 2 \mathrm{~L}(5.25 \mu \mathrm{s})$

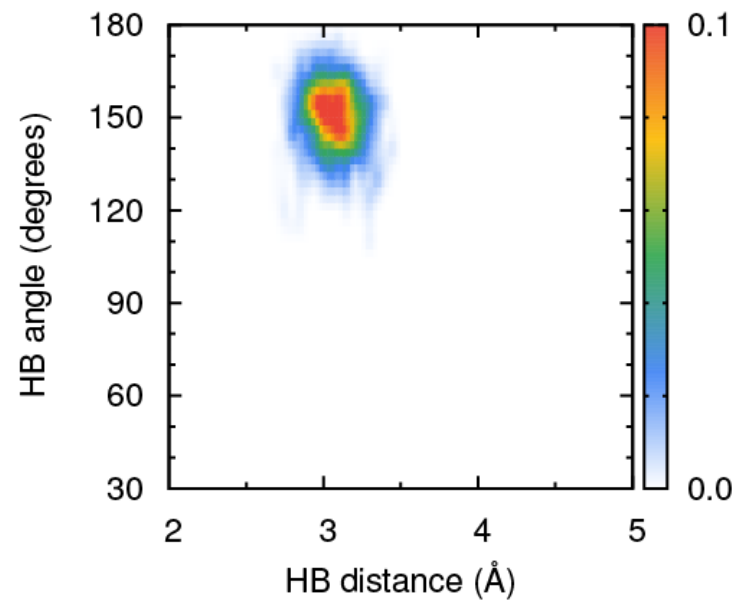

(b) $5 \mathrm{H} 2 \mathrm{~K}(2 \mu \mathrm{s})$

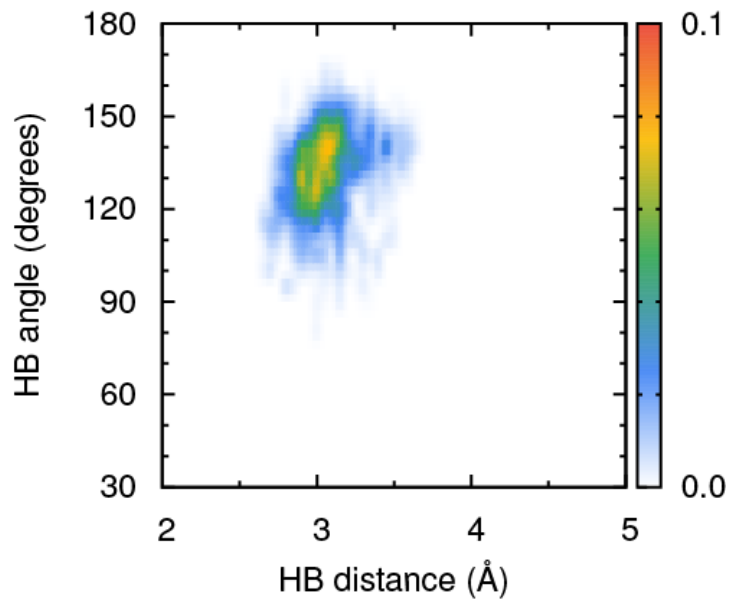

(d) $5 \mathrm{H} 2 \mathrm{M}(13.8 \mu \mathrm{s})$

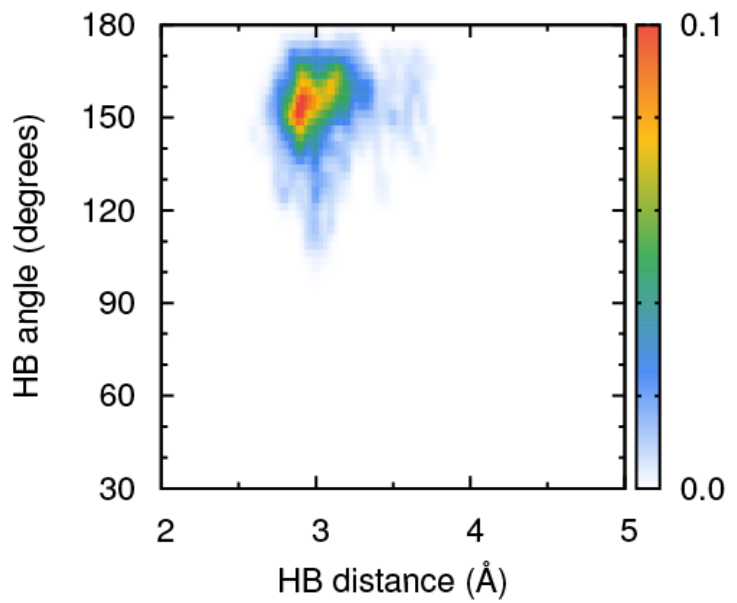

Figure S9. Two-dimensional probability distribution functions for the hydrogen-bond (HB) distance and the HB angle between SB and Wat452 obtained from the last 10-ps trajectories in the 50-ps unbiased DC-DFTB-MD simulations at the initial state of the primary proton transfer in IBRM for (a) 5B6X, (b) 5H2K, (c) 5H2L, and (d) 5H2M. Note that the times in parentheses are the same as those in Figure 1. 
(a) $5 \mathrm{~B} 6 \mathrm{X}$ (760 ns)

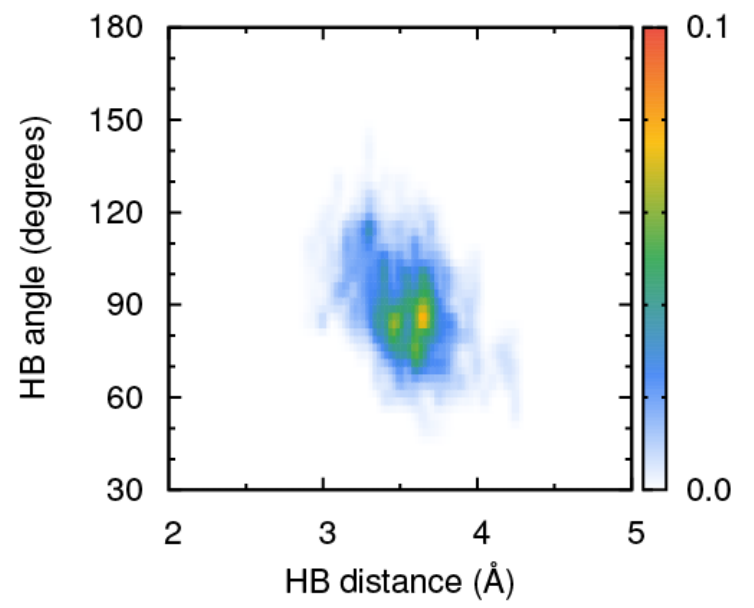

(c) $5 \mathrm{H} 2 \mathrm{~L}(5.25 \mu \mathrm{s})$

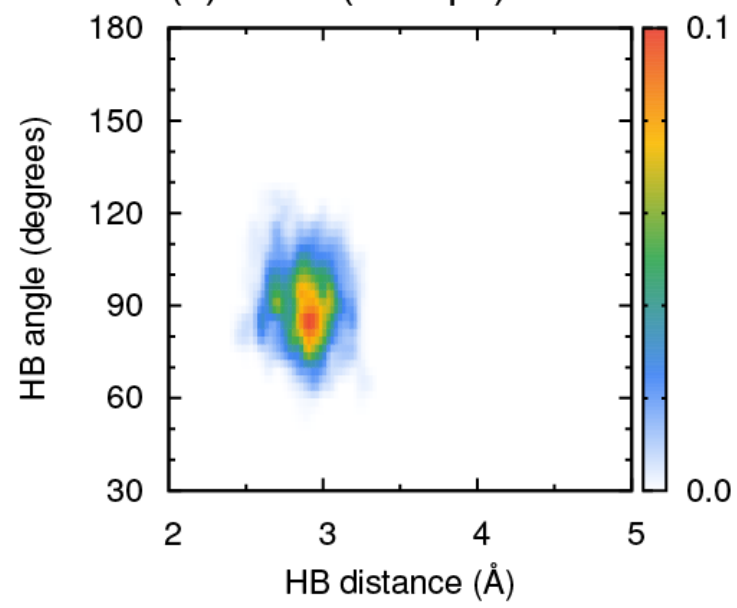

(b) $5 \mathrm{H} 2 \mathrm{~K}(2 \mu \mathrm{s})$

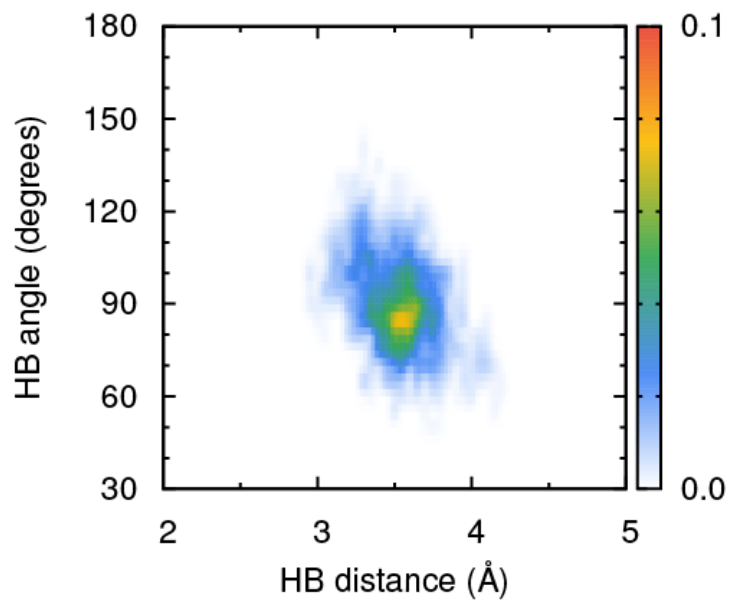

(d) $5 \mathrm{H} 2 \mathrm{M}(13.8 \mu \mathrm{s})$

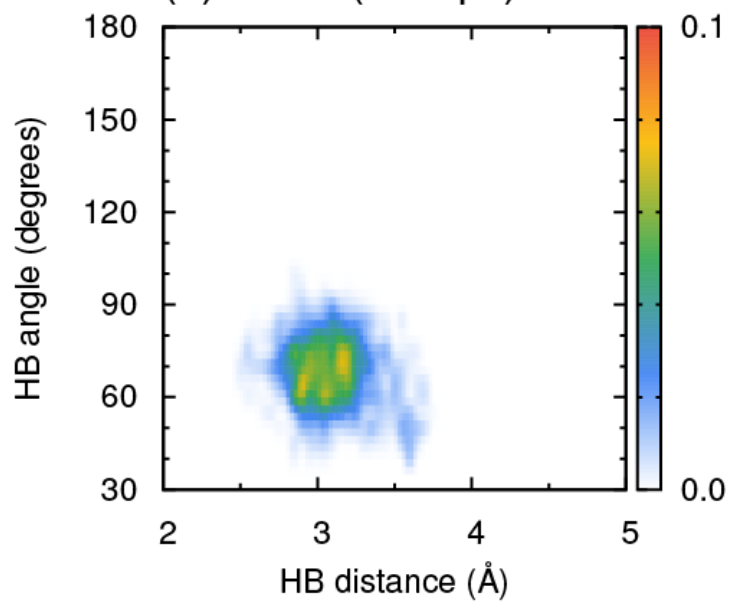

Figure S10. Two-dimensional probability distribution functions for the hydrogen-bond (HB) distance and the HB angle between SB and Thr89 obtained from the last 10-ps trajectories in the 50-ps unbiased DC-DFTB-MD simulations at the initial state of the primary proton transfer in IBRM for (a) 5B6X, (b) 5H2K, (c) 5H2L, and (d) 5H2M. Note that the times in parentheses are the same as those in Figure 1. 
(a) $5 \mathrm{~B} 6 \mathrm{X}$ (760 ns)

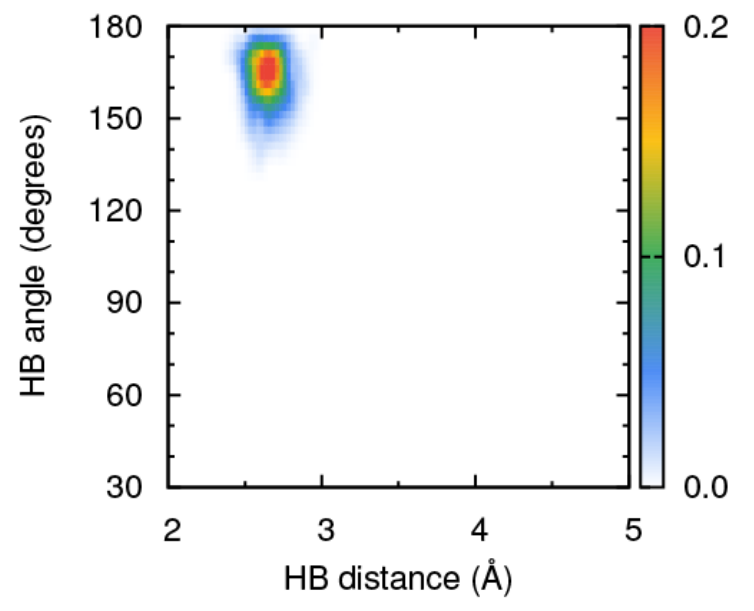

(c) $5 \mathrm{H} 2 \mathrm{~L}(5.25 \mu \mathrm{s})$

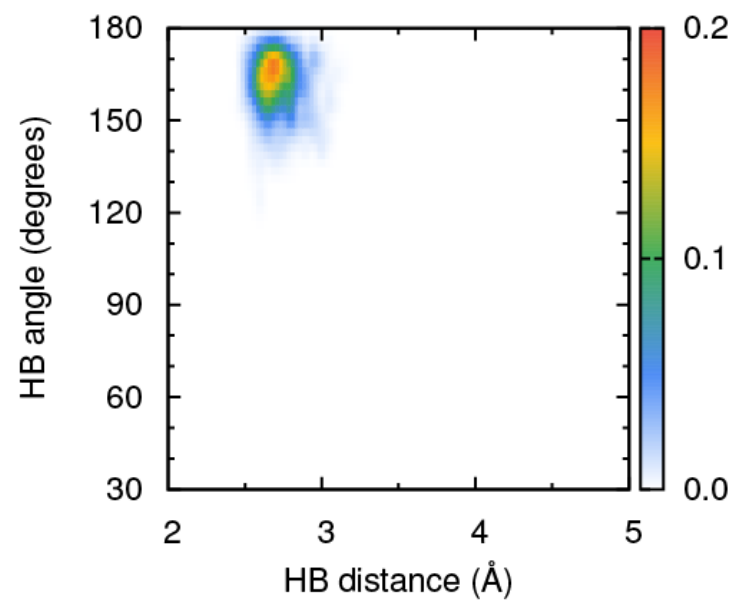

(b) $5 \mathrm{H} 2 \mathrm{~K}(2 \mu \mathrm{s})$

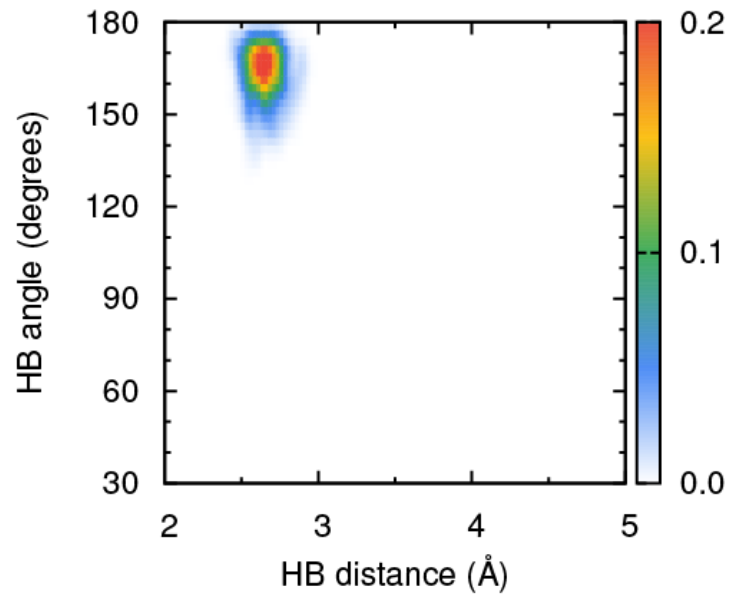

(d) $5 \mathrm{H} 2 \mathrm{M}(13.8 \mu \mathrm{s})$

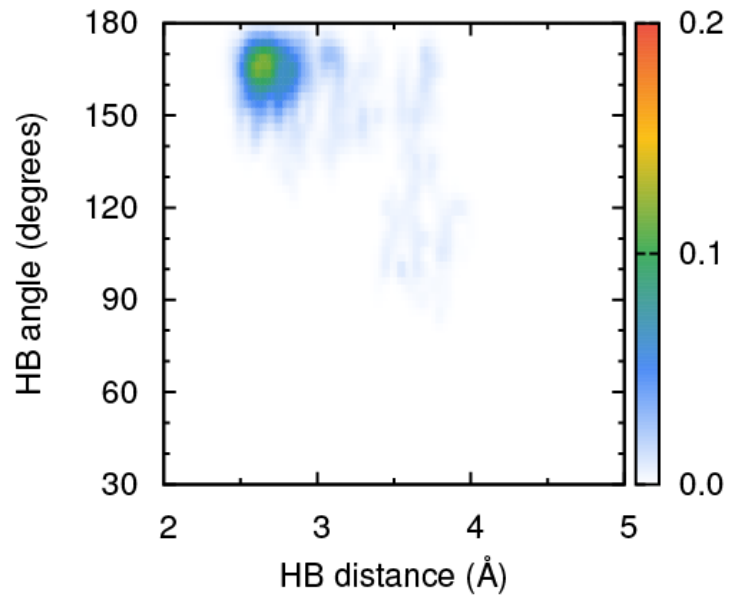

Figure S11. Two-dimensional probability distribution functions for the hydrogen-bond (HB) distance and the HB angle between Thr89 and Asp85 obtained from the last 10-ps trajectories in the 50-ps unbiased DC-DFTB-MD simulations at the initial state of the primary proton transfer in IBRM for (a) 5B6X, (b) 5H2K, (c) 2H2L, and (d) 5H2M. Note that the times in parentheses are the same as those in Figure 1. 
(a) Wat452-Thr89

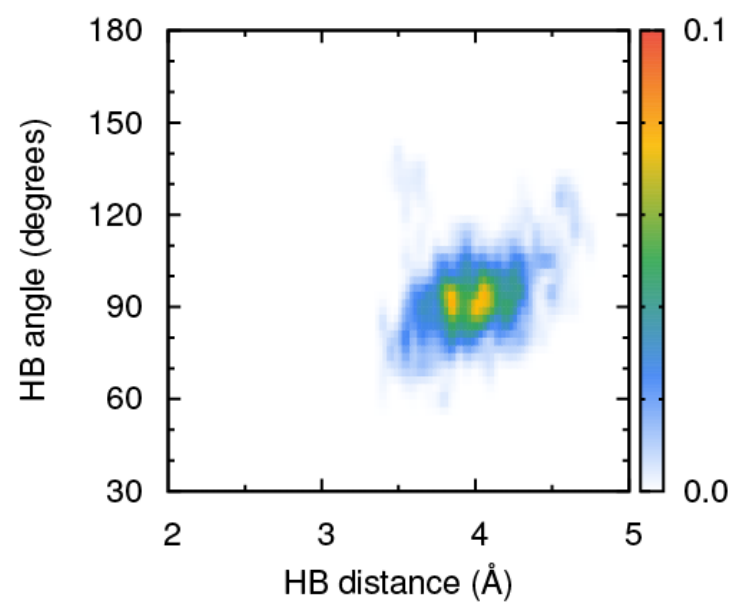

(c) SB-Thr89

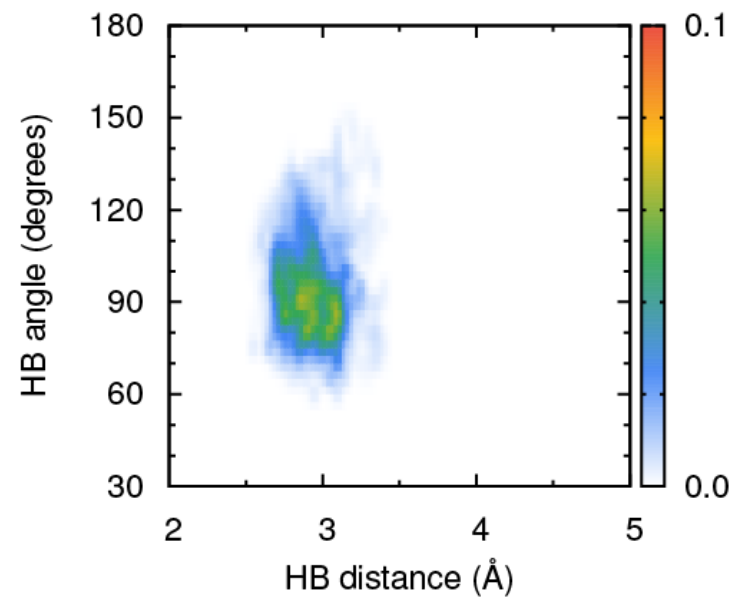

(b) SB-Wat452

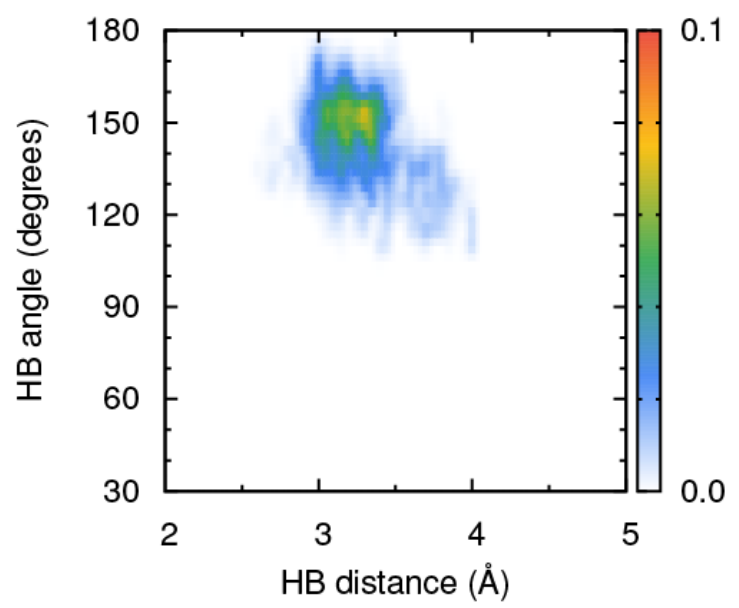

(d) Thr89-Asp85

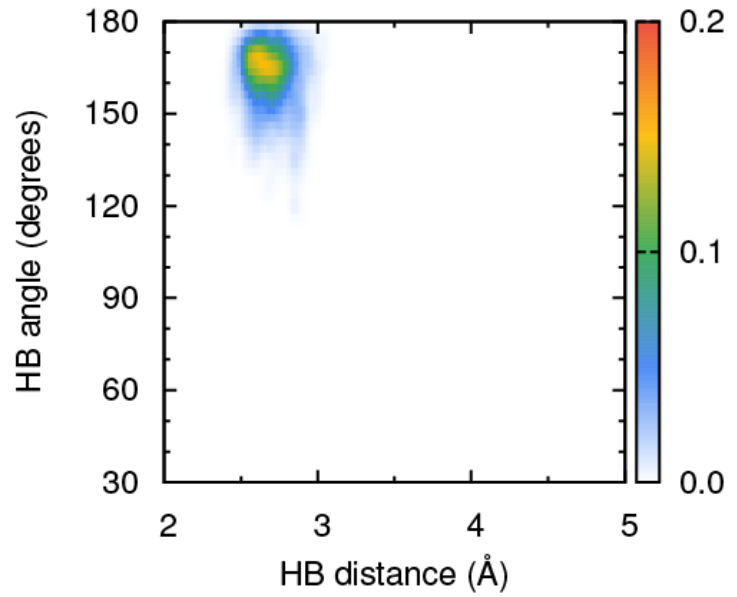

Figure S12. Two-dimensional probability distribution functions for the hydrogen-bond (HB) distances and the HB angles between (a) Wat452 and Thr89, (b) SB and Wat452, (c) SB and Thr89, and (d) Thr89 and Asp85 obtained from the unbiased DC-DFTB-MD simulations for IBRM starting from the 5H2K structure without Wat401. 
(a)

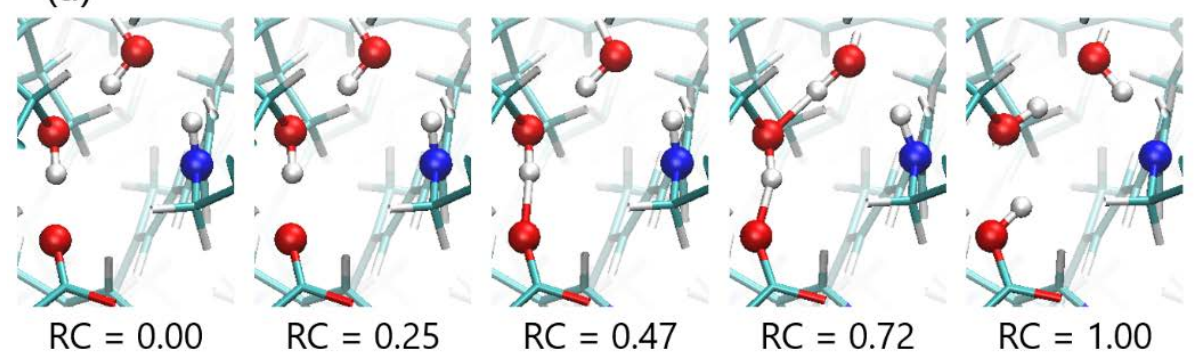

(b)

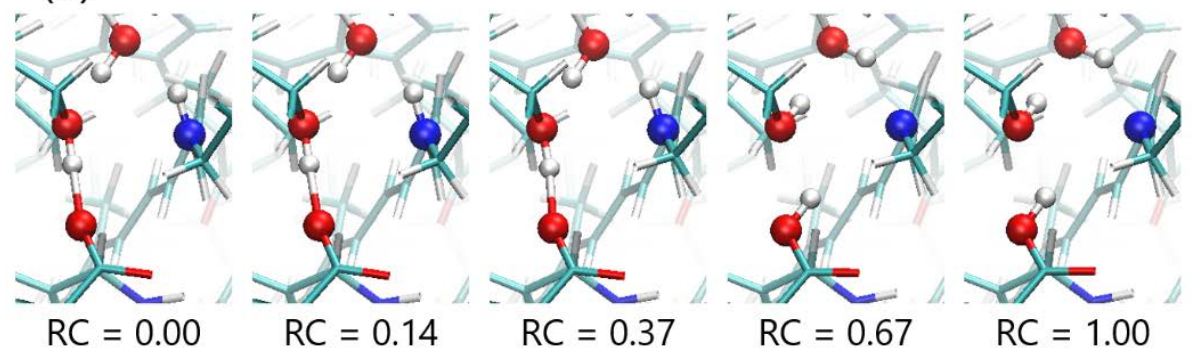

Figure S13. Converged images of the primary proton transfer in (a) IBRM and (b) EBRM models along the MEPs calculated using DC-DFTB climbing image NEB calculation with $N=3$. RC is an abbreviation of reaction coordinate. 
(a)

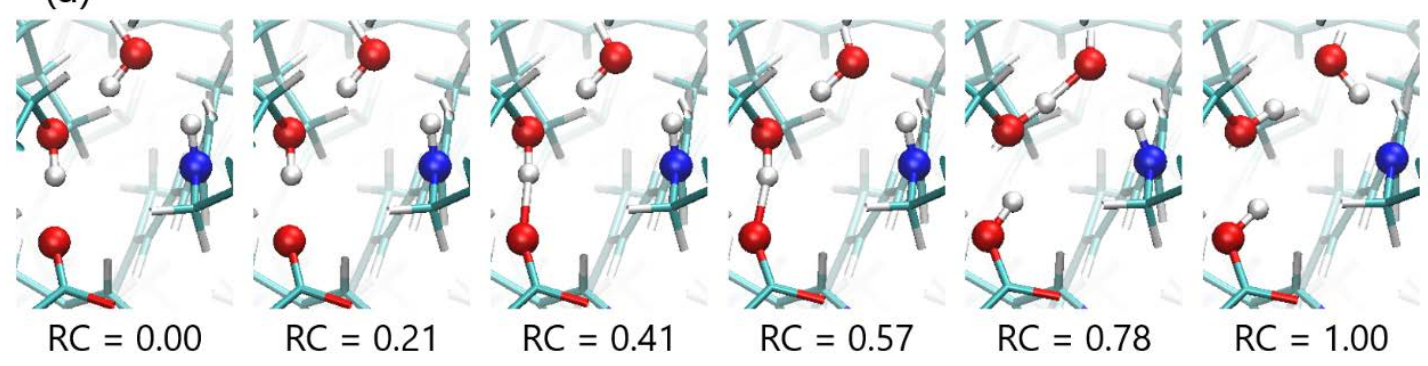

(b)

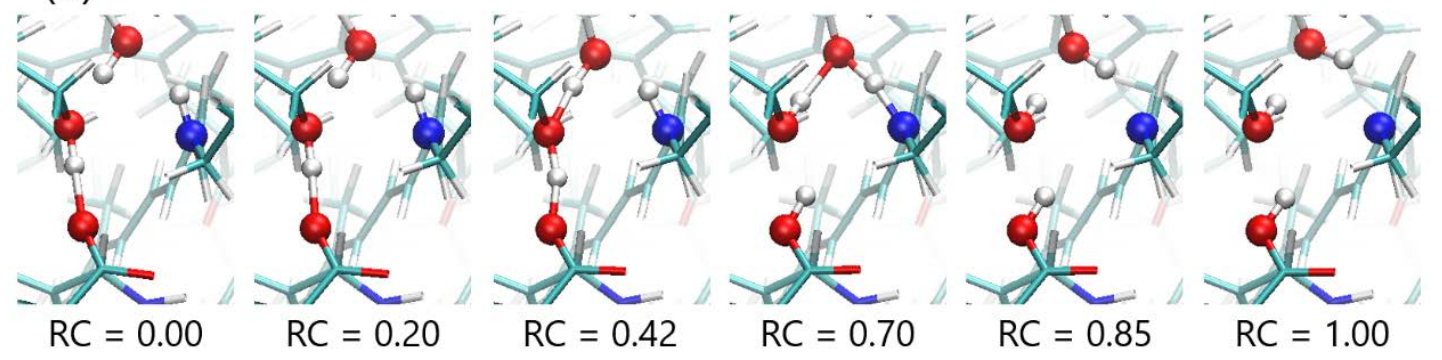

Figure S14. Converged images of the primary proton transfer in (a) IBRM and (b) EBRM models along the MEPs calculated using DC-DFTB climbing image NEB calculation with $N=4$. RC is an abbreviation of reaction coordinate. 
(a)
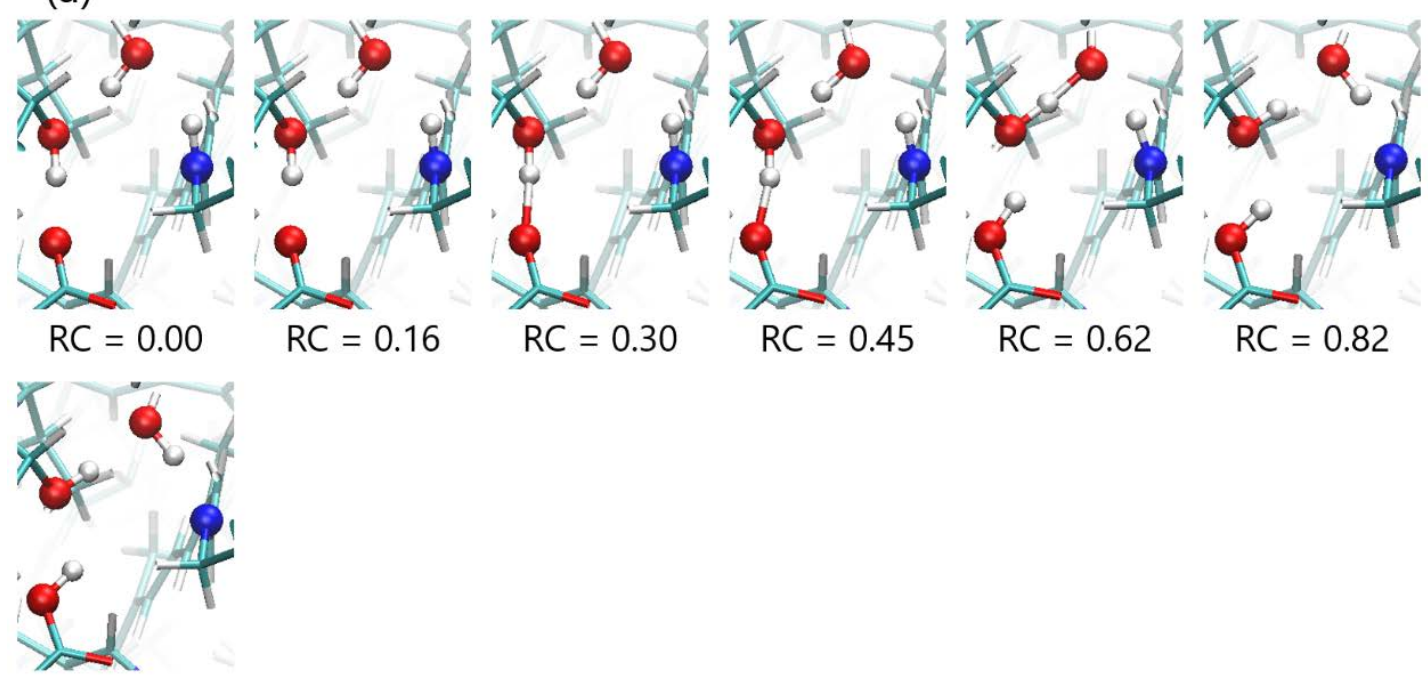

$R C=1.00$

(b)
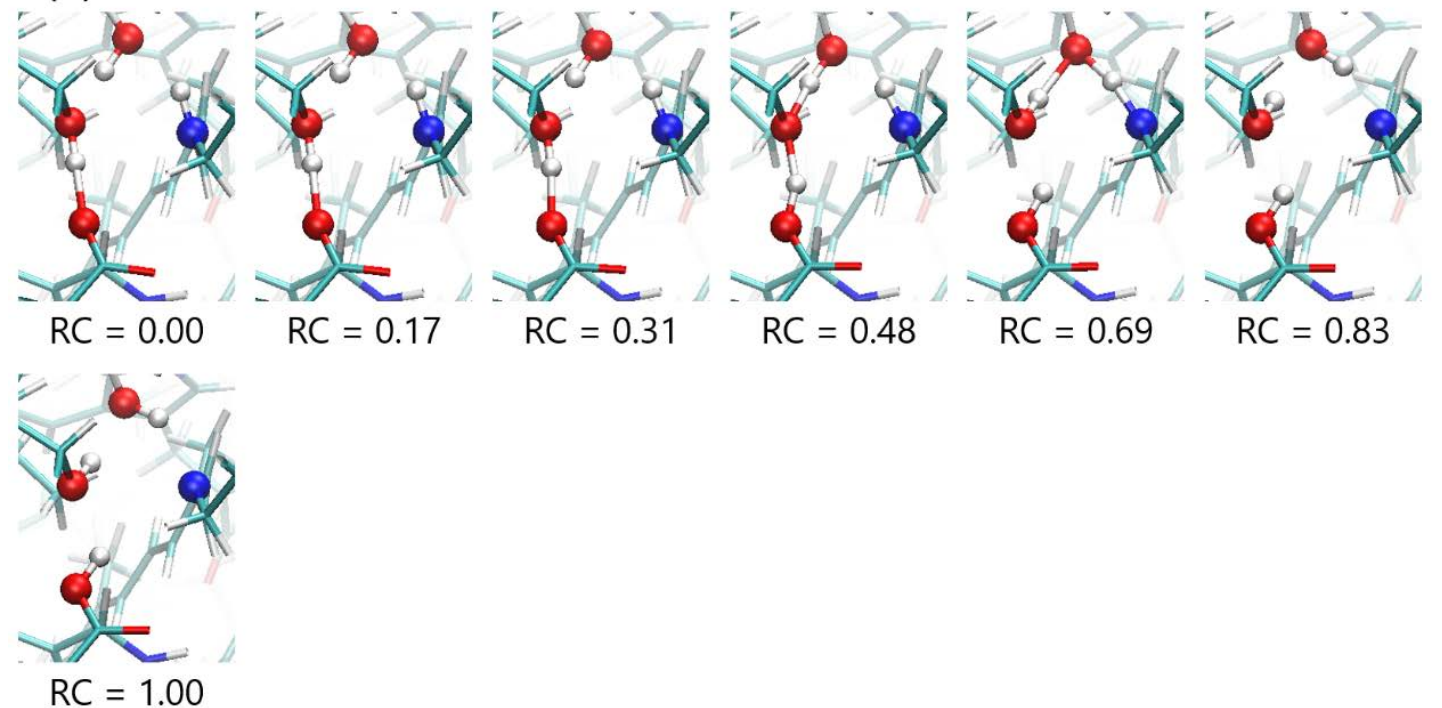

Figure S15. Converged images of the primary proton transfer in (a) IBRM and (b) EBRM models along the MEPs calculated using DC-DFTB climbing image NEB calculation with $N=5$. RC is an abbreviation of reaction coordinate. 
(a)
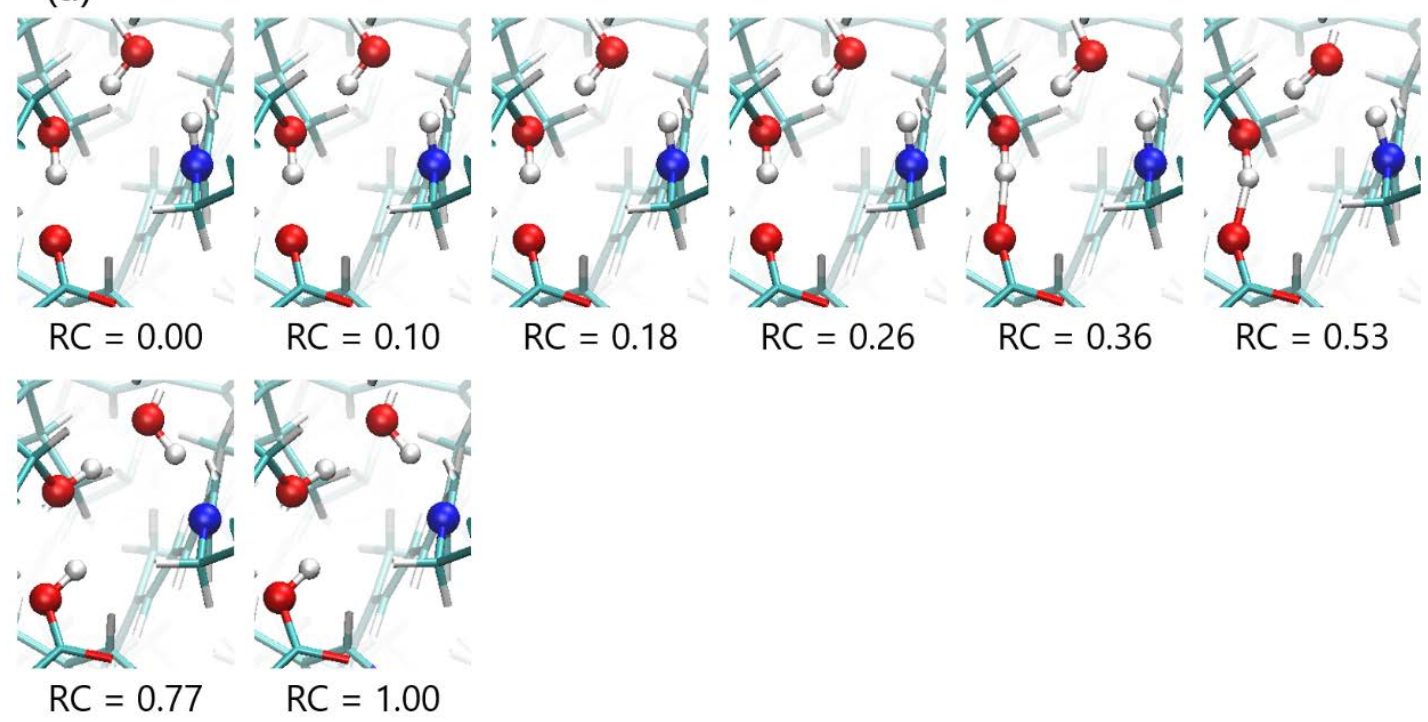

(b)
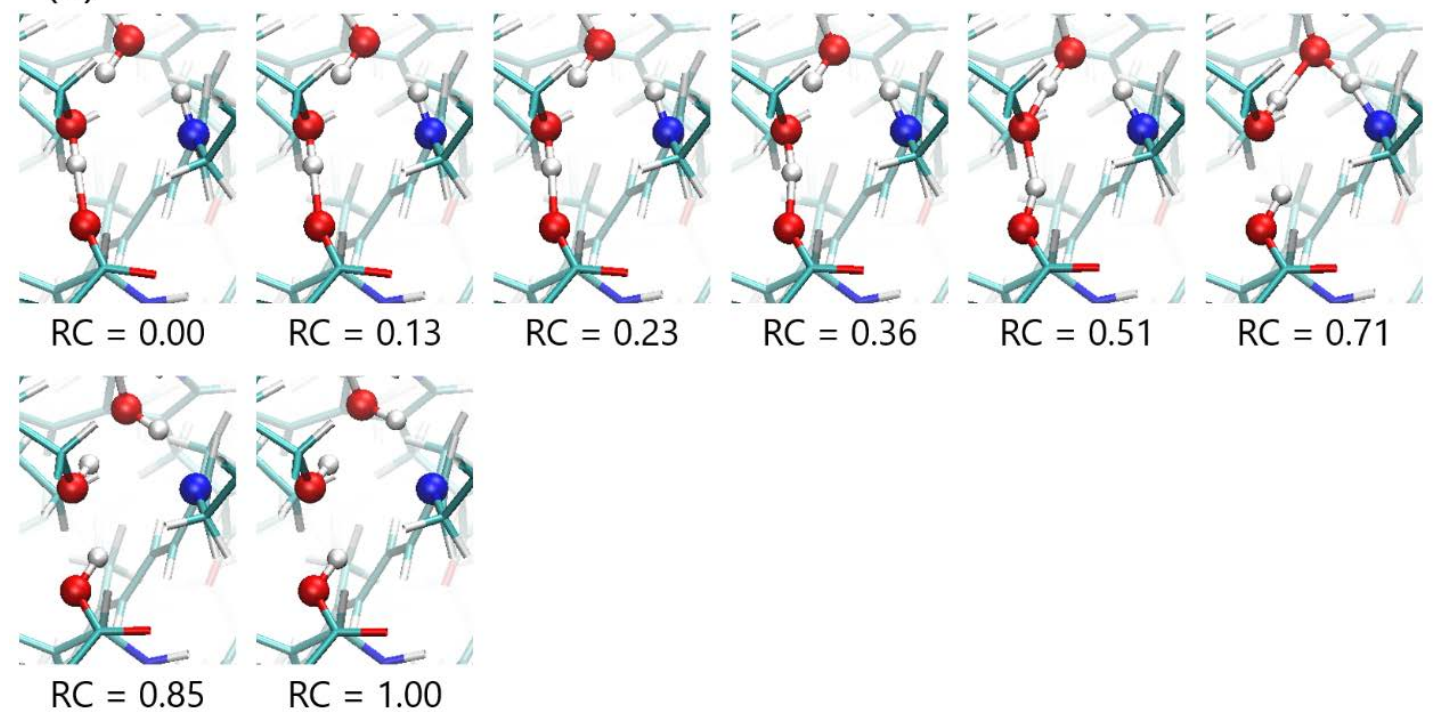

Figure S16. Converged images of the primary proton transfer in (a) IBRM and (b) EBRM models along the MEPs calculated using DC-DFTB climbing image NEB calculation with $N=6$. RC is an abbreviation of reaction coordinate. 
(a)
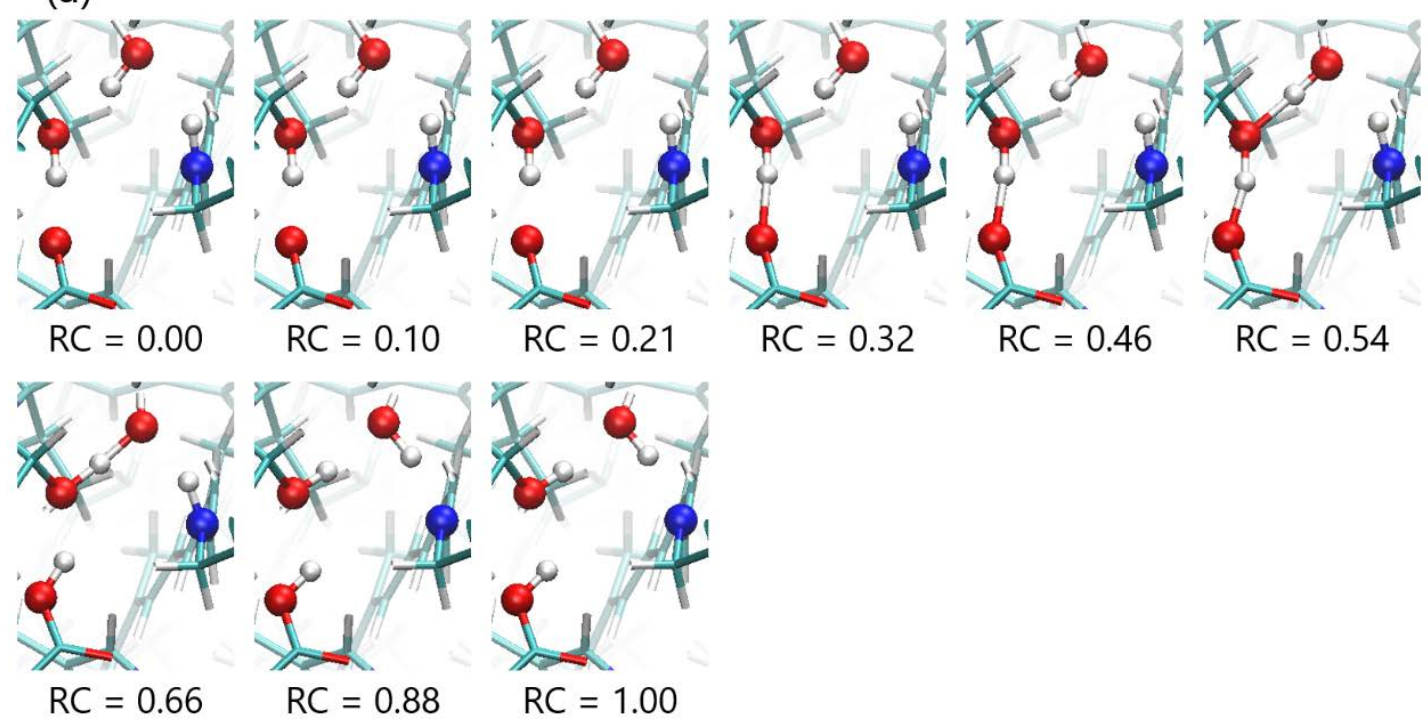

(b)
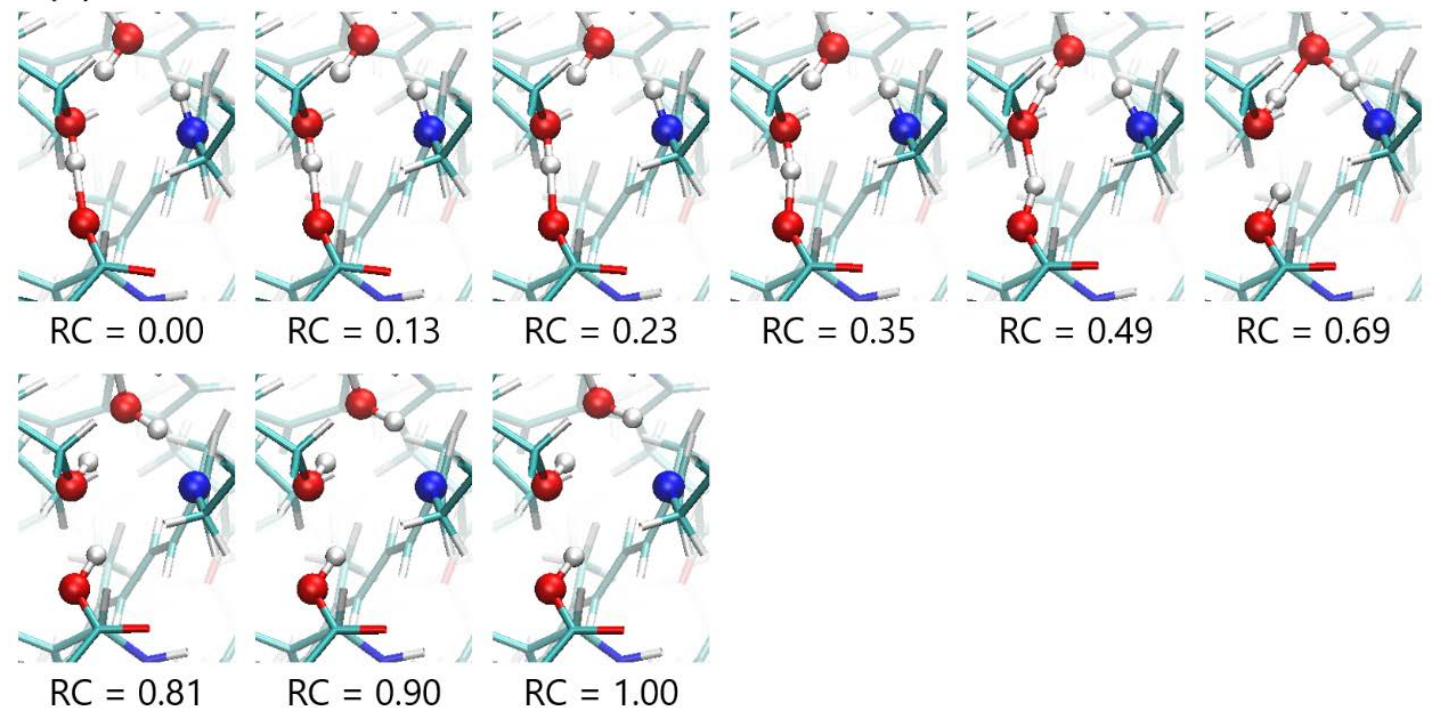

Figure S17. Converged images of the primary proton transfer in (a) IBRM and (b) EBRM models along the MEPs calculated using DC-DFTB climbing image NEB calculation with $N=7$. RC is an abbreviation of reaction coordinate. 
(a)

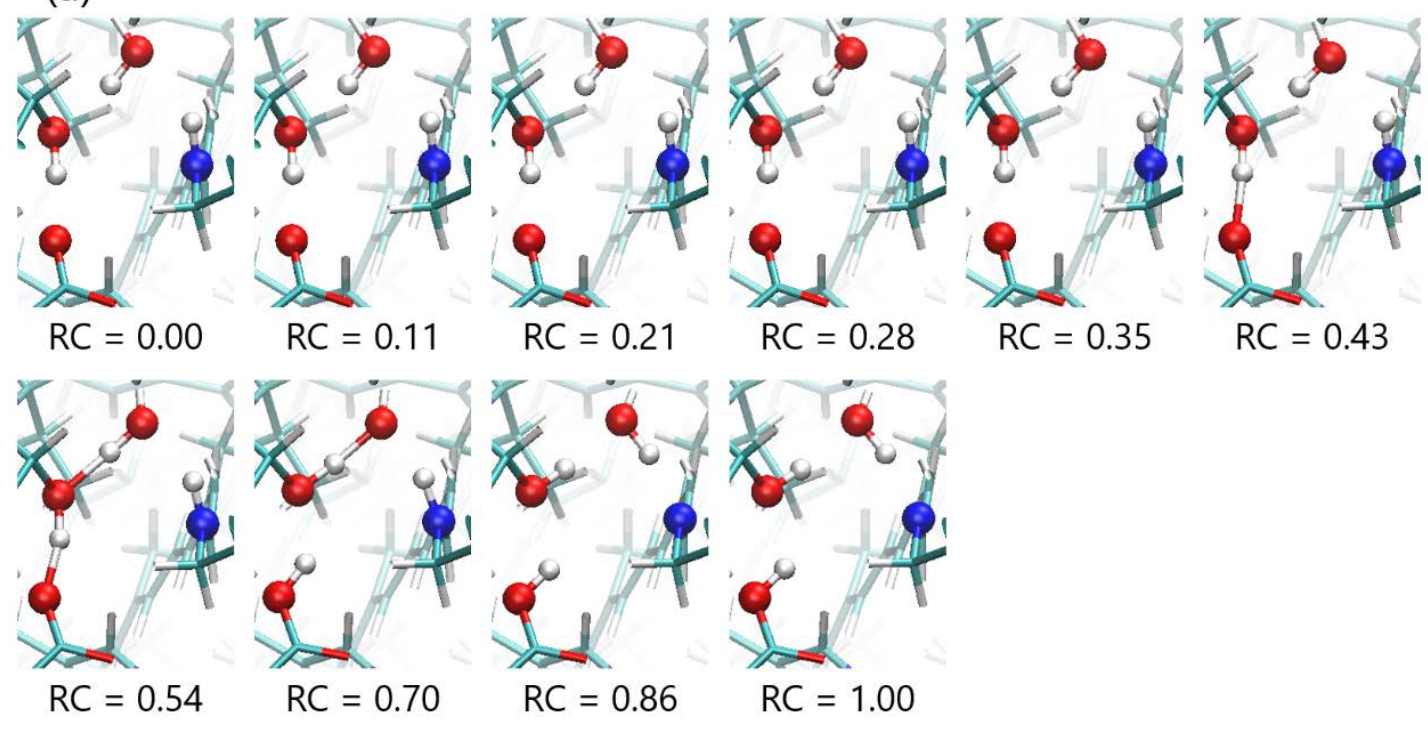

(b)
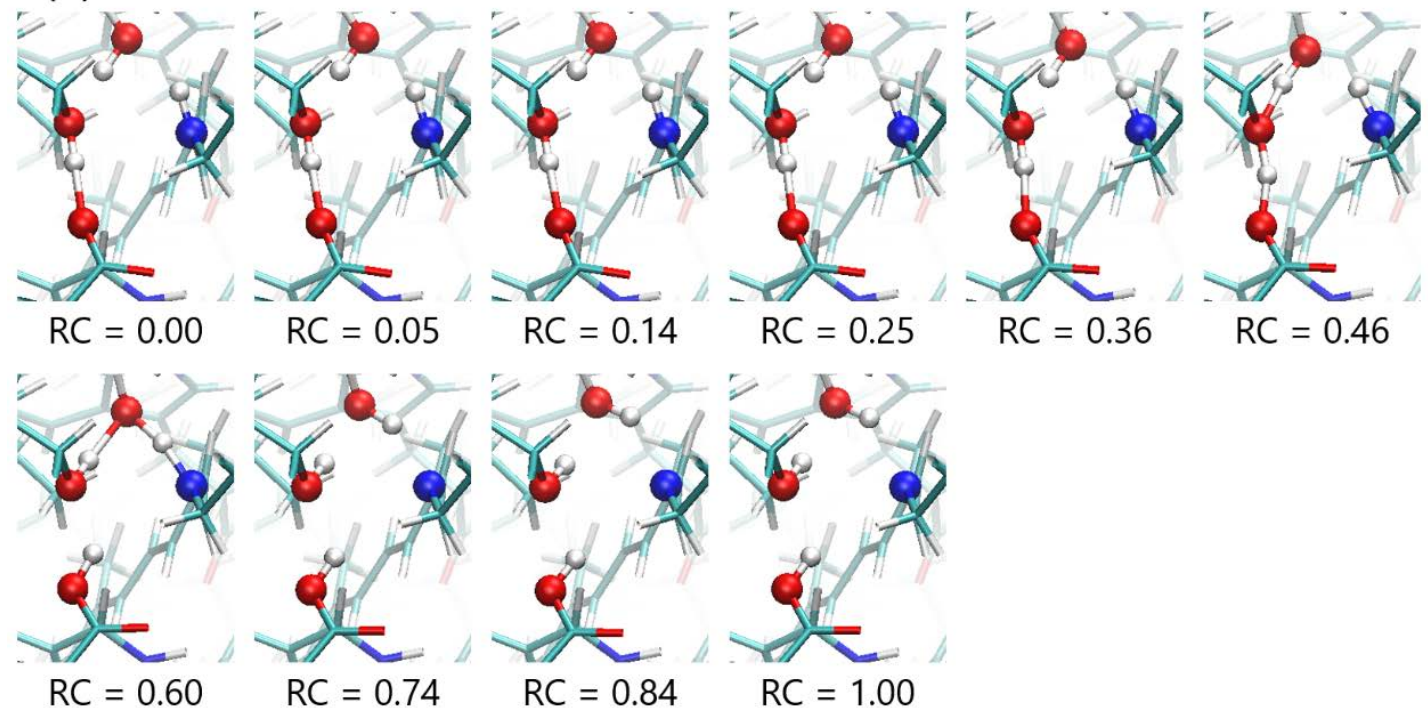

Figure S18. Converged images of the primary proton transfer in (a) IBRM and (b) EBRM models along the MEPs calculated using DC-DFTB climbing image NEB calculation with $N=8$. RC is an abbreviation of reaction coordinate. 


\section{Complete Author List}

45. Nango, E.; Royant, A.; Kubo, M.; Nakane, T.; Wickstrand, C.; Kimura, T.; Tanaka, T.; Tono, K.; Song, C.; Tanaka, R.; Arima, T.; Yamashita, A.; Kobayashi, J.; Hosaka, T.; Mizohata, E.; Nogly, P.; Sugahara, M.; Nam, D.; Nomura, T.; Shimamura, T.; Im, D.; Fujiwara, T.; Yamanaka, Y.; Jeon, B.; Nishizawa, T.; Oda, K.; Fukuda, M.; Andersson, R.; Båth, P.; Dods, R.; Davidsson, J.; Matsuoka, S.; Kawatake, S.; Murata, M.; Nureki, O.; Owada, S.; Kameshima, T.; Hatsui, T.; Joti, Y.; Schertler, G.; Yabashi, M.; Bondar, A.-N.; Standfuss, J.; Neutze, R.; Iwata, S. A three-dimensional movie of structural changes in bacteriorhodopsin. Science. 2016, 354, 1552-1557.

46. Nogly, P.; Weinert, T.; James, D.; Carbajo, S.; Ozerov, D.; Furrer, A.; Gashi, D.; Borin, V.; Skopintsev, P.; Jaeger, K.; Nass, K.; Båth, P.; Bosman, R.; Koglin, J.; Seaberg, M.; Lane, T.; Kekilli, D.; Brünle, S.; Tanaka, T.; Wu, W.; Milne, C.; White, T.; Barty, A.; Weierstall, U.; Panneels, V.; Nango, E.; Iwata, S.; Hunter, M.; Schapiro, I.; Schertler, G.; Neutze, R.; Standfuss, J. Retinal isomerization in bacteriorhodopsin captured by a femtosecond x-ray laser. Science. 2018, 361, eaat0094.

47. Kovacs, G. N.; Colletier, J.-P.; Grünbein, M. L.; Yang, Y.; Stensitzki, T.; Batyuk, A.; Carbajo, S.; Doak, R. B.; Ehrenberg, D.; Foucar, L.; Gasper, R.; Gorel, A.; Hilpert, M.; Kloos, M.; Koglin, J. E.; Reinstein, J.; Roome, C. M.; Schlesinger, R.; Seaberg, M.; Shoeman, R. L.; Stricker, M.; Boutet, S.; Haacke, S.; Heberle, J.; Heyne, K.; Domratcheva, T.; Barends, T. R. M.; Schlichting, I. Threedimensional view of ultrafast dynamics in photoexcited bacteriorhodopsin. Nat. Commun. 2019, 10, 3177.

48. Weinert, T.; Skopintsev, P.; James, D.; Dworkowski, F.; Panepucci, E.; Kekilli, D.; Furrer, A.; Brünle, S.; Mous, S.; Ozerov, D.; Nogly, P.; Wang, M.; Standfuss, J. Proton uptake mechanism in bacteriorhodopsin captured by serial synchrotron crystallography. Science. 2019, 365, 61-65.

65. Zheng, Q.; Miura, S.; Ko, S.; Miyazaki, K.; Watanabe, E.; Okoshi, M.; Chou, C.-P.; Nishimura, Y.; Nakai, H.; Kamiya, T.; Honda, T.; Akikusa, J.; Yamada, Y.; Yamada, A. Sodium- and potassium-hydrate melts containing asymmetric imide anions for high-voltage aqueous batteries. Angew. Chem. Int. Ed. 2019, 58, 14202-14207.

67. Otake, K.; Otsubo, K.; Komatsu, T.; Dekura, S.; Taylor, J. M.; Ikeda, R.; Sugimoto, K.; Fujiwara, A.; Chou, C.-P.; Sakti, A. W.; Nishimura, Y.; Nakai, H.; 
Kitagawa, H. Confined water-mediated high proton conduction in hydrophobic channel of a synthetic nanotube. Nat. Commun. 2020, 11, 843.

82. Case, D. A.; Babin, V.; Berryman, J. T.; Betz, R. M.; Cai, Q.; Cerutti, D. S.; Cheatham III, T. E.; Darden, T. A.; Duke, R. E.; Gohlke, H.; Goetz, A. W.; Gusarov, S.; Homeyer, N.; Janowski, P.; Kaus, J.; Kolossváry, I.; Kovalenko, A.; Lee, T. S.; LeGrand, S.; Luchko, T.; Luo, R.; Madej, B.; Merz, K. M.; Paesani, F.; Roe, D. R.; Roitberg, A.; Sagui, C.; Salomon-Ferrer, R.; Seabra, G.; Simmerling, C. L.; Smith, W.; Swails, J.; Walker, R. C.; Wang, J.; Wolf, R. M.; Wu, X.; Kollman, P. A. AMBER 14, University of California, San Francisco, 2014. 